\title{
Top Quark Mass Measurements at CDF
}

\author{
Pedro A. Movilla Fernandez ${ }^{\mathrm{a}}$ (on behalf of the CDF Collaboration) \\ ${ }^{a}$ Lawrence Berkeley National Laboratory, 1 Cyclotron Road, Berkeley, California 94720, U.S.A.
}

\begin{abstract}
Recent measurements of the mass of the top quark $(t)$ are presented using $162 \mathrm{pb}^{-1}$ of data of $p \bar{p}$ collisions at $\sqrt{s}=1.96 \mathrm{TeV}$ collected by the CDF detector at the Tevatron collider during Run II. The analyses focus on the semi-leptonic decay mode $t \bar{t} \rightarrow(b l \nu)(\bar{b} q \bar{q})$ with one or two identified bottom quarks $(b)$. The Template Method reconstructs the invariant mass of the top quark in each event. The Multivariate Template Method enhances this approach by adding information on the event topology. The Dynamical Likelihood Method discriminates between possible mass values using top quark decay observables and attempts to use the maximum amount of information on top quarks provided by the Standard Model. All three methods produce similar results. The Dynamical Likelihood Method yields a top quark mass of $177.8_{-5.0}^{+4.5}$ (stat) \pm 6.2 (sys) $\mathrm{GeV} / c^{2}$.
\end{abstract}

\section{INTRODUCTION}

The first experimental observation of the top quark $(t)$ by the $\mathrm{CDF}$ and $\mathrm{D} \varnothing$ collaborations at the Tevatron about ten years ago [1] represented an impressive confirmation of the Standard Model (SM) and marked the beginning of a successful top quark physics program at the Tevatron. Properties of the top quark were first investigated using $110 \mathrm{pb}^{-1}$ of data at $\sqrt{s}=1.8 \mathrm{TeV}$ collected in the Run I period between 1992 and 1996. The top quark mass, $m_{t}$, was measured in all decay topologies (e.g. [23]) arising from $p \bar{p} \rightarrow t \bar{t} X$ final states. After a recent reanalysis of D $\varnothing$ Run I data 4, the collaborations updated the combined Run I value to $m_{t}=178.0 \pm 4.3 \mathrm{GeV} / c^{2}[\underline{5}$.

The precise knowledge of $m_{t}$ is of great interest for particle physics. It is a fundamental parameter of the SM, but more importantly, the extremely high mass gives the top quark particular relevance in the calculation of other SM parameters. Electroweak corrections to the $W$ propagator introduce a quadratic dependence on the $W$ boson mass $m_{W}$ and a logarithmic dependence on the mass of the long-hypothesized but still unobserved Higgs boson, $m_{H}$. With the current precision level of $2.5 \%$ for $m_{t}$ and $<0.1 \%$ for $m_{W}$, the most likely value for $m_{H}$ obtained from global SM fits is $m_{H}=114_{-45}^{+69} \mathrm{GeV} / c^{2}$, and the upper $95 \%$ CL limit is $260 \mathrm{GeV} / c^{2}$ [5]. A more accurate determination of $m_{t}$ would provide the opportunity for stringent consistency tests of the SM.

The precision measurement of the top quark mass is therefore a very active topic in the current physics program of CDF in Run II. The upgraded Tevatron complex started in 2001, producing $p \bar{p}$ collisions at $\sqrt{s}=1.96 \mathrm{TeV}$ with steadily increasing instantaneous luminosities up to a record of $\sim 10^{32} \mathrm{~cm}^{-2} \mathrm{~s}^{-1}$ (as of the time of this report). The higher collision energy led to an increase of the SM production $t \bar{t}$ cross-section of $35 \%$ [ The CDF detector [7] underwent substantial improvements in the geometrical acceptance of the tracking and calorimeter systems as well as of the silicon vertex detector which is crucial for the identification of $B$-hadrons from $t$ decays.

All $t \bar{t}$ decay modes are being investigated intensively. Here we report on three recent CDF measurements of $m_{t}$ in the lepton plus jets channel using $162 \mathrm{pb}^{-1}$ data collected between March 2002 and September 2003.

\section{EVENT SELECTION}

According to the Standard Model, top quarks at the Tevatron are mainly produced in pairs through quark-anti-quark annihilation $(85 \%)$ or gluon-gluon fusion (15\%), followed by a prompt decay $t \rightarrow W b$ with a branching ratio $\mathrm{BR} \sim 100 \%$. The subsequent $W$ decays define the event signature. In the decay mode studied here, one $W$ decays into two quarks, and the other $W$ decays into 
an electron $(e)$ or a muon $(\mu)$ and the corresponding neutrino $(\nu)$. This channel has a manageable signal to background ratio $S / B \sim \mathcal{O}(1)$ and a satisfactory $B R \sim \frac{8}{27}$ that is viewed as a good compromise between all available decay modes. ${ }^{1}$

The final state has a spherical topology due to $t \bar{t}$ production near threshold and is characterized by four jets, one charged lepton with high transverse energy $\left(E_{\mathrm{T}}\right)$, and a sizable amount of missing transverse energy $\left(\mathbb{E}_{\mathrm{T}}\right)$ due to the undetectable $\nu$. Top quark candidate events are selected by requiring exactly one isolated $e$ or $\mu$ with $E_{\mathrm{T}}>20 \mathrm{GeV}$ and $E_{\mathrm{T}}>20 \mathrm{GeV}$. Furthermore, at least three jets with $E_{\mathrm{T}}>15 \mathrm{GeV}$ and one jet with $E_{\mathrm{T}}>8 \mathrm{GeV}$ have to be present. The DLM analysis (explained below) requires exactly four jets with $E_{\mathrm{T}}>15 \mathrm{GeV}$. The jets must lie in the pseudo-rapidity region $|\eta|<2$ so that they are well measured by the detector. Finally, one or two displaced secondary vertices (originating from the decay of long-lived $B$-hadrons) are identified by the silicon vertex detector ("b-tags"). This requirement improves the sample purity by a factor of 3-4 compared to the non- $b$-tagged case.

Dominant background sources are $W b \bar{b}$ and $W c \bar{c}$ final states, non- $W$ QCD processes and events where one jet is misidentified as a $b$-jet. Minor sources of background are $W W / W Z, W c$ and single top quark $(t \bar{b})$ events.

\section{TOP MASS MEASUREMENTS}

\subsection{Template Method}

The Template Method (TM) closely follows the traditional mass measurement strategy in Run I. To get the momenta of the daughter particles of the assumed initial $t \bar{t}$ configuration, the raw jet energies are corrected for detector effects and artifacts due to the limited jet cone size, as well as for hadronization and for various non- $t \bar{t}$ contributions of the same event. One part of the corrections are directly derived from the data, another part is derived using a Herwig $t \bar{t}$ Monte Carlo [8] and a simulation of the CDF detector. The analysis treats the four leading $E_{\mathrm{T}}$ jets as quark jets from the $t \bar{t}$ system. The event re-

\footnotetext{
${ }^{1}$ The dilepton mode (with $e$ or $\mu$ ) has $S / B \sim 10, B R \sim \frac{4}{81}$, the all-hadronic mode has $S / B<\frac{1}{10}, B R \sim \frac{4}{9}$.
}

\section{CDF Run II Preliminary (162 $\left.\mathrm{pb}^{-1}\right)$}

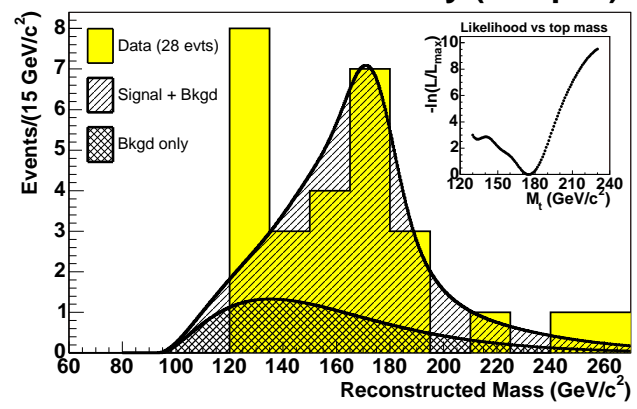

Figure 1. Reconstructed top quark mass data compared with the combined signal plus background fit.

construction is complicated by the combinatorial problem of correctly assigning these jets to the four initial partons, yielding six (two) solutions ("permutations") for the single (double) $b$ tag case. The number of solutions doubles because of the twofold ambiguity of the longitudinal component of the $\nu$ momentum.

For each configuration, an invariant top quark mass is reconstructed by minimizing a $\chi^{2}$ expression calculated using the transverse momenta of the jets, the charged lepton, and the missing energy. The $\chi^{2}$ takes into account the detector resolution as well as the decay widths of the $t$ and the $W$, and also incorporates various kinematic constraints (e.g. $\left.m_{t}=m_{\bar{t}}, M_{W^{+}}=M_{W^{-}}\right)$. The solution with the smallest $\chi^{2}$ value is taken as the "reconstructed top mass" of the candidate event. This quantity is used to build templates for signal Monte Carlo samples with varying generated $m_{t}$ but also for various background samples. The signal templates are parametrized by continuous functions of $m_{t}$. The templates serve as probability densities to be compared with the data in order to derive a top quark mass probability for each event. The most likely value for $m_{t}$ is extracted using an unbinned likelihood fit that maximizes the joint probability density (= product of all event-by-event probability densities) for the whole sample of reconstructed masses. It also includes a background fraction constraint.

With a sample of 28 candidate events (Fig. 1) and $6.8 \pm 1.2$ expected background events 9 the method yields $m_{t}=174.9_{-7.7}^{+7.1}$ (stat) \pm 6.5 (sys) $\mathrm{GeV} / c^{2}$. The systematic error is dominated by uncertainties of the jet energy scale $\left( \pm 6.3 \mathrm{GeV} / c^{2}\right)$ which mainly arises from the cal- 
ibration of the CDF calorimeter to a uniform response and setting the absolute jet energy scale.

\subsection{Multivariate Template Method}

The Multivariate Template Method (MTM) has three main features: 1) The jet energy scale (JES) is adjusted event-by-event. 2) Three types of signal templates are used to handle the combinatorial problem. 3) Other kinematic variables, in addition to $m_{t}$, are used to improve S/B discrimination. The method performs the same generic jet corrections as TM but uses a refined kinematic fitter for the event reconstruction. In particular it includes a JES factor calibrated by the reconstruction of the $W \rightarrow q \bar{q}^{\prime}$ decay using a $W$ mass constraint. On average this partially compensates for systematic JES shifts but also increases the statistical error because of event-byevent fluctuations of the JES. The fitter contains a JES constraint which allows the balance of JES systematics vs. statistical uncertainties. Further kinematic constraints similar to TM are imposed. Only the neutrino solution for which the $t$ and $\bar{t}$ masses are closest is taken.

Three signal templates are used to handle jetparton combinatorics: correct permutation samples, incorrect permutation samples, and incorrect jet assignment samples (where e.g. a gluon jet is assigned to a quark). It can be shown that the knowledge of the event template type improves the mass resolution by a factor of $\sim 1.7$ in an ideal scenario with no background. MTM attempts to predict the correct permutation probability by using the differences between the smallest $\chi^{2}$ and the $\chi^{2}$ values of the other permutations, and by using kinematic information with weak $m_{t}$ dependence, specifically the angle between the lepton and $b$-quark in the $W \rightarrow l \nu$ rest frame, and $t \bar{t}$ spin correlation variables. These probabilities are used to weight the signal templates accordingly.

In addition to the reconstructed top quark mass, further kinematic variables with high S/B separation power are used to construct multidimensional templates. From studies of various histogram divergence measures it turns out that the scalar sum of the transverse momenta of the four leading jets $\left(P_{\mathrm{T} 4}\right)$ is a promising variable to distinguish signal from noise. The templates are

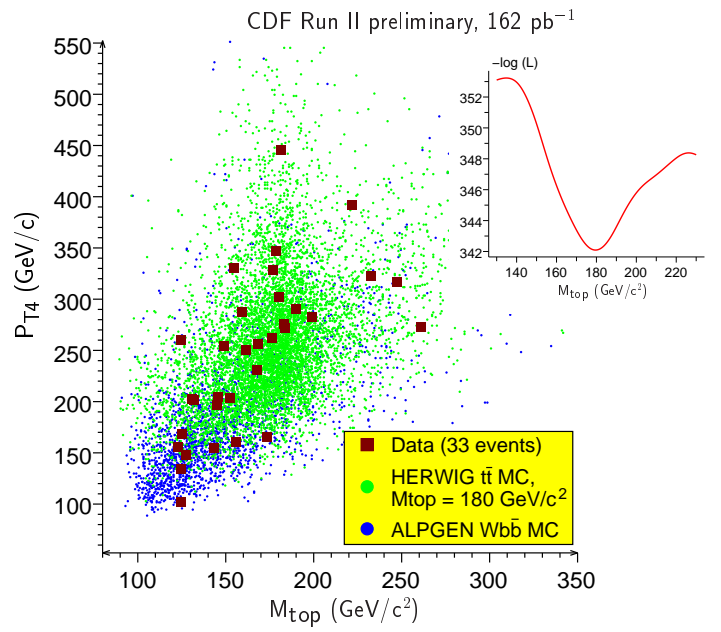

Figure 2. Reconstructed mass vs. $P_{\mathrm{T} 4}$ for the data and for signal and background expectation.

generated using Kernel Density Estimation [10], a non-parametric density reconstruction technique particularly suited for multivariate densities.

The top quark mass likelihood is expressed in terms of multivariate probability densities for signal and background and considers the three types of signal templates. The background fraction is allowed to float freely. The JES constraint is optimized w.r.t. the expected total error. A maximum likelihood method applied to a sample of 33 selected events (Fig. 21) yields $m_{t}=179.6_{-6.3}^{+6.4}($ stat $) \pm 6.8(\mathrm{sys}) \mathrm{GeV} / c^{2}$ and a most probable background fraction of $0.34 \pm 0.14$.

\subsection{Dynamical Likelihood Method}

The Dynamical Likelihood Method (DLM) is an original CDF method 11] that attempts to use the full amount of information about the $t \bar{t}$ process provided by the Standard Model. The top quark mass likelihood $L^{(i)}\left(m_{t}\right)$ for the $i^{t h}$ event is given by

$$
\begin{aligned}
L^{(i)}\left(m_{t}\right)= & \int \sum_{\text {perm }} \sum_{\nu \text { sol. }} \frac{2 \pi^{4}}{\text { flux }}|\mathcal{M}|^{2} F\left(z_{1}, z_{2}\right) f\left(p_{\mathrm{t}}\right) \\
& \times w\left(\mathbf{x}, \mathbf{y} ; m_{t}\right) \mathrm{d} \mathbf{x}
\end{aligned}
$$

where $\mathbf{x}$ denotes the parton momenta and $\mathbf{y}$ denotes jet observables. $F\left(z_{1}, z_{2}\right)$ is the parton distribution function for the incoming partons, and $f\left(p_{\mathrm{t}}\right)$ is the probability that the $t \bar{t}$ system has received a transverse momentum $p_{\mathrm{t}}$ due to initial state radiation. The matrix element $\mathcal{M}$ for 


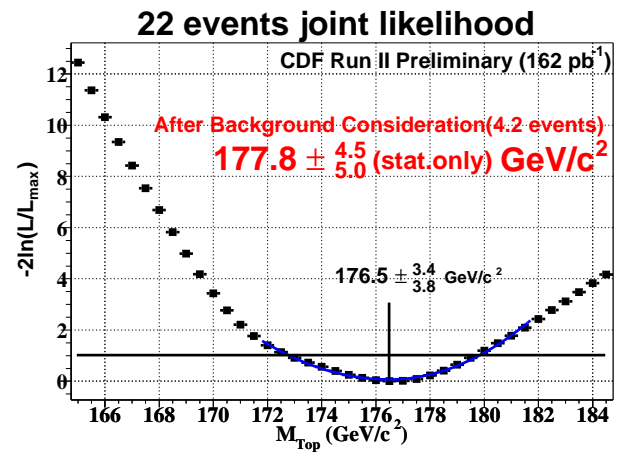

Figure 3. DLM joint negative log likelihood.

the $t \bar{t}$ production and decay process is linked to the four-jet configuration using transfer functions $w\left(\mathbf{x}, \mathbf{y} ; m_{t}\right)(\mathrm{TF})$. These are Bayesian probabilities that a $t \bar{t}$ parton configuration $\mathbf{x}$ with a given $m_{t}$ was generated when $\mathbf{y}$ was reconstructed. The TFs are functions of $\left(E_{\mathrm{T}}^{\mathrm{parton}}-E_{\mathrm{T}}^{\mathrm{jet}}\right) / E_{\mathrm{T}}^{\mathrm{parton}}$ and parametrized in jet $E_{\mathrm{T}}$ and $\eta$ bins. They are calculated for $W$ or $b$ jets separately using a Herwig $t \bar{t} \mathrm{MC}$ for a fixed top quark mass. DLM performs some of the jet corrections as TM and MTM (e.g. relative calorimeter response, calorimeter to particle correction), while it handles the transition from partons to particles via TFs.

The likelihood function $L^{(i)}$ sums all permutations and neutrino solutions. The most likely top quark mass is obtained by maximizing $\Pi_{i} L^{(i)}\left(m_{t}\right)$. The joint negative log likelihood curve extracted from a sample of 22 selected events is shown in Fig. 3] It should be noted that the likelihood considers only the signal but not the background matrix elements. The background contribution is hence minimized by requiring exactly four jets. Residual background effects, but also the top quark mass dependence of the TFs, are taken into account by applying an MC-based correction ("mapping" function) to the maximum likelihood mass. Using $4.2 \pm 0.2$ expected background events, this method yields $m_{t}=177.8_{-5.0}^{+4.5}$ (stat) \pm 6.2 (sys) $\mathrm{GeV} / c^{2}$.

\section{SUMMARY AND OUTLOOK}

Three recent CDF measurements of the top quark mass have been performed in the $t \bar{t}$ lepton plus jets channel using $162 \mathrm{pb}^{-1}$ of Tevatron Run II data. The results are $m_{t}=$
$174.9_{-7.7}^{+7.1}$ (stat) $\pm 6.5(\mathrm{sys}) \mathrm{GeV} / c^{2}(\mathrm{TM}), m_{t}=$ $179.6_{-6.3}^{+6.4}$ (stat) \pm 6.8 (sys) $\mathrm{GeV} / c^{2}$ (MTM), and $m_{t}=177.8_{-5.0}^{+4.5}$ (stat) $\pm 6.2($ sys $) \mathrm{GeV} / c^{2}$ (DLM).$^{2}$ The systematic error is dominated by jet energy uncertainties. MTM allows for an optimal compromise between statistical and systematic errors and will be more powerful at higher accumulated luminosity. The DLM result has a considerably less statistical uncertainty than the other methods and is competitive with the best CDF Run I measurement [2].

Tevatron is performing well and is expected to provide $400 \mathrm{pb}^{-1}$ by the end of $2004,1 \mathrm{fb}^{-1}$ by the end of 2005 , and $4.4-8.5 \mathrm{fb}^{-1}$ by the end of Run II, thus leading to a significant improvement of the statistical error. A priority is the reduction of the jet energy systematics. The stated $\mathrm{CDF}$ goal for the total error is $3 \mathrm{GeV} / c^{2}$. Substantial progress is expected soon, e.g. due to recent developments in the calorimeter simulation. Furthermore there is a substantial potential to refine the analyses. Through these measurements, CDF has established techniques that will provide a precise determination of the top quark mass in the future.

\section{REFERENCES}

1. F. Abe et al., Phys. Rev. Lett. 74 (1995) 2626; S. Abachi et al., Phys. Rev. Lett. 74 (1995) 2632.

2. T. Affolder et al., Phys. Rev. D 63 (2001) 032003.

3. B. Abbott et al., Phys. Rev. D 58 (1998) 052001; S. Abachi et al., Phys. Rev. Lett. 79 (1997) 1197.

4. V.M. Abazov et al., Nature 429 (2004) 638.

5. Tevatron EWG, hep-ex/0404010 LEP EWG, Phys. Lett. B 565 (2003) 61; updated Aug 2004: http://lepewwg . web.cern.ch/LEPEWWG/

6. N. Kidonakis and R. Vogt, Phys. Rev. D 68 (2003) 114014.

7. CDF Coll., FERMILAB-PUB-96-390-E (1996).

8. G. Corcella et al., JHEP 0101 (2001) 10.

9. CDF Coll., P. Merkel, these proceedings.

10. D. Scott, Multivariate Density Estimation, WileyInterscience, 1992.

11. K. Kondo et al., J. Phys. Soc. Jap. 57 (1988) 4126 ;

\footnotetext{
${ }^{2}$ The results are consistent with an updated CDF measurement in the dilepton channel reported after this conference: $m_{t}=176.5_{-16.0}^{+17.2}$ (stat) \pm 6.9 (sys) $\mathrm{GeV} / c^{2}$.
} 